\title{
Análisis de Validez y Confiabilidad de una Escala para la Evaluación de Estilos Decisorios Operativos.
}

\author{
Susana Celeste Azzollini* \& Pablo Domingo Depaula** \\ * Consejo Nacional de Investigaciones Científicas y Técnicas; Instituto de Investigaciones en Psicología de la \\ Universidad de Buenos Aires.Buenos Aires, Argentina. **Centro de Investigaciones Sociales y Humanas para la \\ Defensa; Instituto Universitario del Ejército. Buenos Aires, Argentina.
}

\begin{abstract}
Resumen. En el marco de una investigación experimental con el objetivo de analizar la influencia de la memoria autobiográfica y la inteligencia cultural sobre el tipo de toma de decisiones (analíticas o intuitivas) en contextos caracterizados por la diversidad cultural y la incertidumbre, se adaptó la Encuesta de Conocimiento Tácito (Daake, Dawley, $\&$ Anthony, 2004) resultando en una Escala de Toma de Decisiones Operativas (ETDO) para medir el estilo predominante en la toma de decisiones. Este instrumento fue administrado a una muestra probabilística al azar de 272 voluntarios asignados a misiones de paz. El objetivo del presente trabajo es validar dicha escala y determinar su confiabilidad. Los análisis estadísticos se realizaron con el software SPSS 15.0.

$\mathrm{El}$ instrumento presenta aceptables propiedades psicométricas, alcanzando todos ítems saturaciones factoriales elevadas en alguno de los cinco componentes relevados. El alfa de Cronbach muestra una aceptable consistencia interna.

Palabras clave: Validación; Toma de decisiones; Misiones de paz.
\end{abstract}

\section{Analysis of the validity and reliability of a scale for assessment of operational decision-making styles.}

\begin{abstract}
Within the framework of an experimental investigation with the objective to analyze the influence of the autobiographical memory and cultural intelligence on the type of decision making (analytical or intuitive) in contexts characterized by the cultural diversity and the uncertainty, we adapted the Survey of Tacit Knowledge (Daake, Dawley \& Anthony, 2004) result to a Scale of Operative Decision Styles (SODS) in order to measure the predominant style in the decision making. This instrument was administered to a probabilistic sample of 272 volunteers assigned to peace missions. The objective of the present work is to validate this scale and to determine its confidence. The statistical analyses were made with the SPSS 15.0 software.

The instrument presents acceptable psychometric properties, displaying all items elevated factorial saturations in all the five released components. The alpha of Cronbach shows a passable internal consistency.
\end{abstract}

Key Words: Validation; Decision making; Missions of peace.

\section{Introducción}

El estudio de la toma de decisiones constituye un área de gran interés para la psicología cognitiva, organizacional, educacional, entre otras disciplinas. Daake, Dawley y Antony (2004) argumentan que determinadas investigaciones precedentes han abordado las teorías y los procesos de toma de decisiones, ocupándose de distinguir de manera tajante las decisiones analíticas de las intuitivas (e.g. Simon, 1955), atribuyendo a esta visión dicotómica 
diferentes modos de procesamiento (Epstein, 1994; Evans, 2008).

Aludiendo a los procedimientos preestablecidos para la toma de decisiones y la resolución de problemas, gran parte de la literatura científica coincide en que la información formal constituye la materia prima para la toma de decisiones analíticas. Sin embargo, algunos autores argumentan que a menudo ésta cuenta con ciertas deficiencias, tales como ser limitada cuantitativa y/o cualitativamente, demasiado generalizada y poco fiable para el decisor; bajo determinadas circunstancias contextuales es recibida tardíamente, al tiempo que la rigidez de los objetivos planificados puede fomentar el uso de información inadecuada, entre otras dificultades encontradas (Daake et al., 2004).

Los hallazgos de Simon $(1947,1982)$ vinculados al concepto de racionalidad limitada por una vasta concurrencia de posibilidades ambientales de las que depende la toma de decisiones, muestran que la intuición (involucrada en una estrategia emergente) corresponde a un análisis cristalizado en hábito y en capacidad para responder rápidamente por medio de un patrón de reconocimiento (e.g., Klein, 1998; 2003; Simon, 1947; 1955; 1987), resultando así un proceso eficaz para tomar decisiones en contextos complejos e inciertos (Azzollini, Depaula, Piñeyro, \& Torres, 2012).

Mintzberg (1973) le ha dado importancia a la incorporación o integración de decisiones analíticas e intuitivas en la educación para el management, argumentando que en tales procesos emergen operaciones que implican palabras y números, o bien imágenes y sentimientos. Existen enfoques que apoyan la aplicación de un modelo de toma de decisiones sustentado en un modo de pensar holístico-analítico (Nisbett, Peng, Choi, \& Norenzayan, 2001); tal enfoque integral demanda por un lado, imparcialidad, objetividad, focalización sobre los atributos, e implica por otro, orientación hacia el contexto como un todo, atendiendo a las relaciones entre el objeto y el contexto.

En la actualidad, algunos estudios empíricos indagan acerca de la efectividad alcanzada frente a determinadas tareas a través de procesos analíticos o intuitivos, asumiendo que circunstancialmente las decisiones intuitivas pueden arrojar un mayor nivel de optimización en los resultados en contraste con el pensamiento racional (Aczel, Lukacs, Komlos, \& Aitken, 2011; Dijksterhuis \& Nordgren, 2006). 
Estilos cognitivos para la toma de decisiones: Algunos enfoques teóricos, estudios antecedentes y actuales acerca de su evaluación psicológica

Los estilos cognitivos conducen a las personas a adoptar similares actitudes y conductas en una variedad de dominios. Según Raffaldi, Iannello, Vittani y Antonietti (2012), acordando con otros autores (e.g., Riding \& Rayner, 1998), los estilos cognitivos para la toma de decisiones revisten cierta permanencia o estabilidad singular a través del tiempo y frente a diferentes situaciones, distinguiéndose conforme a la consideración de los decisores por los detalles de la situación o la percepción global de la misma. Ellos explican que los individuos analíticos focalizan su atención interesándose en operaciones y procedimientos o en la manera apropiada de hacer las cosas, prefieren la planificación paso a paso y su pensamiento es controlado y dirigido de forma consciente. En contrapartida, los individuos globales tienden a leer rápidamente (scan) la situación formando impresiones globales y permitiendo la inclusión de sus sensaciones o sentimientos en el proceso de toma de decisiones.

Los estilos de toma de decisiones son evaluados mediante instrumentos de autoreporte, aunque algunos autores reconocen la dificultad de estos cuestionarios para describir este tipo de constructos (Aiken, 2002; Gregory, 2007 citados por Raffaldi et al., 2012) vinculados a estilos o tipologías, como también a procesos psicológicos básicos; en efecto, argumentan que los sujetos procuran efectuar un balance entre diferentes tipos de situaciones sobre las cuales eligen y deciden qué hacer en función de los casos que más fácilmente acceden a su mente mientras responden al cuestionario (Raffaldi et al., 2012). Pero en general, los autores coinciden en que las personas intuitivas generalmente incluyen reacciones afectivas en sus elecciones decisorias, mientras que las personas deliberadas o analíticas se abstienen de hacerlo (Betsch \& Kunz, 2008; Raffaldi et al., 2012).

Epstein (1994) afirma que existen dos sistemas paralelos de procesamiento de la información, uno de tipo analítico y otro de tipo intuitivo. Sustentada en tal premisa, la cognitive-experiential self-theory (CETS; Epstein, 2007 citado por Betsch \& Kunz, 2008) conceptualiza a la intuición como un sistema que, de forma automática, aprende de la experiencia, constituyendo el sistema experiencial (Sistema 1) asociado íntimamente al afecto, el que consolida un proceso de pensamiento espontáneo, rápido y en paralelo, cuyo input proviene de los conocimientos almacenados en la memoria de largo plazo (MLP). En contraste, el sistema cognitivo (Sistema 2) corresponde a un proceso de análisis lento, serial, que demanda un mayor nivel de esfuerzo mental, siendo monitoreado y controlado de forma 
deliberada, relativamente flexible y subordinado a reglas lógico-matemáticas (Kahneman, 2003 citado por Betsch y Kunz, 2008).

Betsch y Kunz (2008) afirman que varios inventarios o pruebas psicométricas evalúan las diferencias de estilos cognitivos en la medida en que las personas se inclinan de manera estable hacia el pensamiento intuitivo o deliberado, operacionalizándolos a través de los constructos que aporta la teoría cognitiva-experiencial, por ejemplo. En tal sentido empírico, estos autores destacan la aplicabilidad de un instrumento de evaluación denominado Preference for Intuition and Deliberation Scale (PID; Betsch, 2004, 2007), el que surge de conceptualizar a la intuición como un modo o modalidad decisoria básica, que hace uso del afecto como un criterio decisorio y a las impresiones que surgen instantáneamente; por otro lado, la deliberación es definida como una modalidad decisoria basada en la evaluación explícita, las cogniciones o creencias y el razonamiento. Este test presenta 18 ítems que integran dos dimensiones o sub-escalas bipolares independientes, las que miden el nivel de estabilidad en términos de preferencia por alguno de los dos estilos mencionados (Betsch \& Kunz, 2008).

Por otro lado, el SOLAT questionnaire (Torrance, 1987) intenta medir diferencias individuales en términos de prevalencia de maneras de pensar (Mintzberg, 1991). Una versión italiana de este cuestionario (Antonietti, Fabio, Boari, \& Bonanomi, 2005), ofrece 28 ítems que evalúan el pensamiento derecho (right thinking), de naturaleza intuitiva, el pensamiento izquierdo (left thinking), de tipo analítico, y el pensamiento integrado (integrated thinking), que indica el balance entre las características de ambas formas de pensamiento.

En lo que respecta a la toma de decisiones estratégicas, Daake et al. (2004) consideran la relevancia de la intuición y el conocimiento tácito cuando la incertidumbre o turbulencia ambiental vierte en la mente de los decisores una gran cantidad de información, que en ocasiones, da origen a decisiones estratégicas no programadas; por ello, argumentan que los procesos y equipos de planificación estratégica utilizan combinadamente información racional e intuitiva. Para testear tales premisas, estos autores llevaron a cabo una investigación longitudinal en el ámbito organizacional relativo a la industria del cuidado de la salud, analizando los procesos de planeamiento estratégico de un importante hospital sin fines de lucro situado en el sudeste de Estados Unidos, el que a través de la labor de su Alta Dirección (Chief Executive Officer, CEO), reflexiona sobre los intereses de diversos grupos 
comunitarios. Este estudio se desarrolló a lo largo de siete años a través de varias fases de abordaje cuali-cuantitativo utilizando entrevistas, focus groups, encuestas de análisis de las fortalezas, oportunidades, debilidades y amenazas (FODA) de la institución, sesiones de planeamiento estratégico, y el desarrollo de un instrumento psicométrico de evaluación del conocimiento tácito (sentido común) y de la intuición desplegados en la toma de decisiones estratégicas.

El instrumento Tacit Knowledge Survey (Daake et al., 2004) fue administrado en 21 equipos de planificación estratégica (strategic planning team; SPT), veinte meses después de las sesiones iniciales de planeamiento. Constituye un cuestionario de auto-reporte que los investigadores administraron en una prueba piloto a estudiantes de grado universitario, y en una investigación previa a una muestra de 97 managers, alcanzando un aceptable nivel de consistencia interna $(\alpha=.74)$. Este test dispone de 19 ítems valorables en una escala tipo Likert de cinco opciones de respuesta (1 "fuertemente en desacuerdo" a 5 "fuertemente de acuerdo"), cuyos reactivos se agrupan en dos dimensiones que recopilan datos relacionados con la confianza sobre el conocimiento tácito y el uso de fuentes de información formal. Los autores de esta prueba han sugerido realizar estudios multi-organizacionales para generalizar sus resultados y validar sus conclusiones.

Una técnica psicométrica muy reconocida y ampliamente utilizada en el área clínica, educacional, en procesos de selección de personal, desarrollo de carrera, etc., lo representa el Myers-Briggs Type Indicator (MTBI; Briggs Myers \& Myers, 1980), el que operacionaliza la teoría de los tipos psicológicos propuesta por Jung (1964) instrumentando dos procesos perceptivos (la sensación y la intuición) y dos procesos judicativos (el pensamiento y el sentimiento), que se modifican dando lugar a dos pares de actitudes: extroversiónintraversión y percepción-juicio. Considerando este enfoque, el MBTI evalúa tales actitudes y las preferencias individuales (Pritchard, 2009); por ejemplo, en un reciente estudio realizado sobre una muestra de 214 estudiantes de nivel secundario y universitario que residían en Estambul (Atay, 2012), se estandarizó la Forma M del MBTI (93 ítems) traduciéndolo al idioma turco para analizar su equivalencia lingüística y cultural, y testear ciertas asociaciones con variables demográficas; los resultados evidenciaron una adecuada confiabilidad para la escala global $(\alpha=.819)$, lo mismo para sus cuatro dimensiones, al tiempo que un análisis factorial exploratorio extrajo una solución factorial que explica el $69.73 \%$ de la varianza total. 
Basándose en el modelo teórico de Johnson (1978), quien conceptualiza diferentes estilos de toma de decisiones en base a dos dimensiones bipolares (una de reunión de la información -espontáneo/sistemático- y otra de análisis de la información -interna/externa-), Hardin y Leong (2004) analizaron el Decision Making Inventory (DMI; Coscarelli, 1983 citado por Hardin \& Leong, 1983) seleccionando una muestra de 207 estudiantes (autoidentificados como no-hispánicos) de una universidad de occidente medio de Estados Unidos, los que realizaban un curso introductorio de psicología. El DMI consiste en 20 ítems que describen varias conductas y se responden en una escala Likert de seis puntos (1 "nunca" a 7 "siempre"). Los resultados indicaron que no existen diferencias según el género de los sujetos evaluados, a excepción de la dimensión de estilo externo, en la que las mujeres alcanzaron puntajes superiores a los puntajes de los hombres; las confiabilidades fueron bajas, sobre todo para la dimensión de estilo espontáneo $(\alpha=.40 ; \alpha=.23$, tanto para las mujeres como para los hombres), lo que los autores suponen se debe a que tres de las cuatro sub-escalas estuvieron compuestas de tan solo tres ítems cada una.

Smith (2006), estudiando el despliegue de la intuición en enfermeros novatos y expertos, asume que las dificultades en el uso y en la medición de este proceso pueden sortearse a través del análisis de información cualitativa y cuantitativa permitiendo su correcta exploración empírica. Cualitativamente, propone abordar a la intuición como "un proceso no-lineal de conocimiento percibido” (p. 83), a través de tres aspectos categóricos, a saber: la conciencia emocional (emotional awareness) -analizada a través de categorías emergentes (grounded theory), análisis de contenido, etnografía, entre otras técnicas-; la conciencia física (physical awareness) y el establecimiento de conexiones (making connections). Cuantitativamente, reconoce la importancia de los test psicométricos, de forma tal que considerando las tres categorías intuitivas mencionadas, Smith (2003, Smith, Thurkettle, \& dela Cruz, 2004) desarrolló un instrumento denominado Use of Intuition by Nursing Students Scale (UINSS) que consta de 33 ítems (dispuestos de un formato escalar tipo Likert de cinco opciones de respuesta -1 "nunca" a 5 "siempre"-), cuyo contenido fue establecido por el juicio de expertos y validado en una muestra de 349 estudiantes de enfermería realizando un análisis de componentes principales, del cual extrajeron siete factores que explicaban el $66.2 \%$ de la varianza y valores de confiabilidad de entre .69 y .89 para la escala general y para cada dimensión; los factores extraídos fueron sensaciones físicas (physical sensations), premoniciones (premonitions), conexiones espirituales (spiritual 
connections), lectura de señales (reading cues), sensaciones de energía (sensing energy), aprehensión (apprehension) y sentimientos tranquilizadores (reassuring feelings).

El UINSS, desarrollado por Smith et al. (2004), fue recientemente validado por Demir et al. (2012) traduciendo el instrumento original al idioma turco en una escala Likert integrada por 25 ítems, de cuyo análisis de componentes principales se obtuvieron cuatro factores que explicaban el $58.51 \%$ de la varianza con una adecuada confiabilidad general $(\alpha=$ .86). Coincidiendo con otros autores (Antonietti et al., 2005; Raffaldi, et al., 2012), no encontraron correlaciones significativas entre la edad de los estudiantes que integraban la muestra bajo estudio y los puntajes alcanzados para la escala total y para sus dimensiones respectivas. Por último, los autores observaron que, dado que los factores obtenidos en su estudio difieren en número a los obtenidos por Smith et al. (2004), la capacidad de tomar decisiones intuitivas diverge en la percepción individual dependiendo de variables culturales y étnicas, de los tipos de sistemas o instituciones y de las diferencias que existen en las currículas de formación profesional de los decisores; tales observaciones, sugieren la construcción y adaptación de escalas de medición de la intuición para diferentes culturas y países, dado que escasean en la literatura científica (Demir et al., 2012).

Otra de las pruebas utilizadas para los estilos de toma de decisiones corresponde al General Decision-Making Style Inventory (GDMSI; Scott \& Bruce, 1995; Curseu \& Schruijer, 2012), el que evalúa cinco estilos decisorios diferentes, el racional, el intuitivo, el dependiente, el evitativo y el espontáneo. Curseu y Schruijer (2012) administraron a una muestra de 102 managers de nivel medio, los 25 ítems del GDMSI y 10 tareas decisorias modificadas de Curseu (2006) para evaluar grados de racionalidad e indecisividad. Analizando las propiedades psicométricas de este inventario, los autores argumentaron que los cinco estilos decisorios (especialmente el estilo racional) no mostraron asociaciones significativas con resultados decisorios (en términos de efectividad de la performance) ni con otras variables relevantes.

\section{Objetivos}

1. Adaptar una prueba psicométrica destinada a la evaluación de los estilos predominantemente desplegados en los procesos de toma de decisiones operativas.

2. Calcular la validez de constructo y la confiabilidad del instrumento. 


\section{Método}

\section{Participantes}

272 militares argentinos que durante el semestre posterior a la evaluación psicométrica participarían voluntariamente de una misión de paz bajo el mandato de la Organización de las Naciones Unidas (ONU). Los sujetos fueron seleccionados a través de un muestreo probabilístico al azar simple, y al momento de desarrollar el estudio se hallaban recibiendo entrenamiento en el Centro Argentino de Entrenamiento Conjunto para Operaciones de Paz (CAECOPAZ), previo a su partida a la misión en la que participarían. El 95.6\% de los participantes eran de sexo masculino $(n=260 ; N=272)$, mientras que el $4.4 \%$ eran de sexo femenino $(n=12 ; N=272$ ); la media de edad (medida en años) fue de 30.32 ( $d t$ $=5.885$ ). Por último, en cuanto a su experiencia en operaciones de paz, el $47.1 \%$ de la muestra había participado previamente de al menos una misión de paz $(n=128 ; N=272)$, mientras que el 52.9\% no había participado de ninguna misión de paz $(n=144 ; N=272)$.

\section{Instrumento}

En el marco de una investigación experimental ${ }^{\mathrm{i}}$ que tuvo como objetivo principal analizar la influencia de la memoria autobiográfica y la inteligencia cultural sobre el tipo de toma de decisiones (analíticas o intuitivas) en contextos caracterizados por la diversidad cultural y la incertidumbre, se elaboró la Escala de Toma de Decisiones Operativas (ETDO), resultante de efectuar la adaptación de la Encuesta de Conocimiento Tácito (Daake et al., 2004) para la medición del estilo predominante en la toma de decisiones.

Preliminarmente, se efectuó la traducción de los ítems originales del idioma inglés al castellano utilizando la técnica de traducción directa. El instrumento resultante, corresponde a una técnica de rendimiento típico cuyos estímulos o reactivos se presentan bajo la forma de juicios o afirmaciones mediante una escala con formato tipo Likert de 5 opciones de respuesta, cuyo escalamiento presenta las categorías "Muy en desacuerdo" (1 punto), "En parte en desacuerdo" ( 2 puntos), "Ni de acuerdo ni en desacuerdo" (3 puntos), "En parte de acuerdo" (4 puntos) y "Muy de acuerdo" (5 puntos). Este instrumento se responde utilizando lápiz y papel, requiriendo entre cinco y diez minutos para leer la consigna y completarlo.

Vale destacar que la adaptación mencionada, y en especial la modificación de la 
denominación original del test, se debió a que los investigadores intentaron desarrollar una técnica cuyos reactivos operacionalicen aspectos de la intuición y/o el análisis deliberado desplegados en procesos decisorios netamente operativos, es decir tareas o procedimientos de nivel operacional y táctico, diferentes al nivel estratégico testeado en la versión de Daake et al. (2004). Para ello, se eliminaron aquellos contenidos de los ítems originales que remitían a decisiones o procedimientos estratégicos, intentando enfatizar en cuestiones operacionales que en general no alcanzan los estratos superiores de la conducción organizacional, dado que en tales casos, las decisiones son tomadas eventualmente en el ambiente o contexto de acción donde se desempeñan los cascos azules en las diversas operaciones de paz. Bajo tales criterios, los decisores pueden operar a través de decisiones analíticas determinadas en mayor medida por el background de conocimientos representados por la doctrina militar y las Normas de Empeñamiento de Naciones Unidas (NN.UU) que rigen reglamentariamente en el área o región donde éstos operan (e.g., Haití, Chipre, etc.), o bien desplegar decisiones espontáneas, altamente arraigadas en componentes emocionales y en la experiencia previa de los sujetos; en este sentido, la adaptación de una escala que originalmente evaluaba decisiones estratégicas justificaba su elección, sobre todo por la utilización del contenido semántico asociado a los recursos o elementos racionales como las reglas, cifras numéricas y otros aspectos estrictamente normativos que emergen de los reglamentos militares.

Finalmente, la escala fue administrada a la muestra definitiva junto a otros instrumentos estandarizados, los cuales fueron contrabalanceados.

\section{Procedimiento}

Como se adelantó en el párrafo anterior, inicialmente se tradujeron del idioma inglés al castellano los reactivos correspondientes a la Encuesta de Conocimiento Tácito (Daake et al., 2004) que originalmente constaba de 19 ítems auto-administrables mediante la modalidad individual. En el curso de una fase preliminar a la administración de los instrumentos, la escala fue sometida a una prueba piloto $(n=10)$ a fin de verificar que las afirmaciones resultaran de fácil compresión, para posteriormente ser administrada a la muestra seleccionada; el instrumento contaba con los 19 ítems originales, cuyas frases el sujeto debía tratar de valorar en función de sus preferencias, de acuerdo a la escala Likert de cinco posiciones, ya descripta. 


\section{Análisis de datos}

Una vez concluida la administración de la batería de instrumentos, se llevó a cabo un análisis factorial exploratorio. Tal análisis estadístico fue realizado a través del soporte técnico del software SPSS 15.0.

\section{Resultados}

A continuación, se presentan los resultados arrojados por sucesivos análisis de componentes principales con rotación Promax. En un primer análisis, se obtuvo una solución factorial cuya medida de adecuación muestral de Kaiser-Meyer-Olkin (KMO) correspondía a .675, indicando que resultaba adecuado efectuar el análisis factorial con los ítems del instrumento, es decir que la matriz de datos era factorizable; de los resultados arrojados por la prueba de esfericidad de Bartlett, se interpretó que las correlaciones de los ítems probablemente no fueran producto del azar sino se deban a la existencia de factores latentes extraídos $\left(X^{2}=799.658 ; g l=171 ; p=.000\right)$.

Se obtuvieron siete factores que explicaban el $60.284 \%$ de la varianza acumulada, pero en la matriz de configuración se observaron saturaciones factoriales positivas en un mismo factor (el quinto) de los ítems 7 (.699) y 18 (.684), pese a que el contenido conceptual de ambos ítems fuera contradictorio en relación a los procesos decisorios que implicaban; el ítem 7 refería a aspectos decisorios propios del estilo intuitivo (Algunas veces me sorprendo de mí mismo frente a la manera en que sé, intuitivamente, las decisiones importantes que tengo que tomar), mientras que el ítem 18 refería a aspectos analíticos (Cuando tomo una decisión en el trabajo, generalmente confío más en hechos concretos que en mi propio sentido común). Por otro lado, el ítem 15 (Creo que la mayoría de las decisiones importantes, no pueden ser resueltas usando el sentido común sino que deben ser planificadas), si bien presentaba una elevada carga factorial (.866), era el único ítem que cargaba en el sexto factor de la solución, mientras que el ítem 19 (Cuando tomo una decisión, usualmente no tengo demasiada información sobre el problema), saturaba de forma positiva en el primer factor (.479) y de forma negativa en el sexto factor (-.483).

Producto de las anteriores observaciones, los investigadores decidieron realizar un nuevo análisis de componentes principales con rotación Promax, excluyendo los ítems 7, 18, 15 y 19. Los resultados arrojados por este análisis evidenciaron una solución de cinco factores que explicaban el 55.538\% de la varianza total $\left(K M O=.650 ; X^{2}=560.031 ; \mathrm{gl}=\right.$ 
105; $p=.000$ ). Analizando la matriz de configuración, se decide eliminar el ítem 6 (.616) (Yo gasto una gran cantidad de tiempo buscando hechos relacionados, cifras e información antes de tomar la mayoría de las decisiones) observando que su contenido no coincidía conceptualmente con los tres ítems restantes que cargaban en el mismo factor (ítems 5, $10 \mathrm{y}$ 9). Asimismo, se observa que el ítem 8 (Con frecuencia, tomo importantes decisiones sin recurrir ampliamente al uso de reportes escritos, documentos normativos, hechos previos, cifras e información concreta) y el ítem16 (Confiando demasiado en hechos y cifras, a menudo las decisiones resultan inadecuadas o poco realistas), presentando ambos saturaciones factoriales en el quinto factor, no referían a una decisión o valoración sobre el propio desempeño sino más bien sobre un concepto general. Pese a esta observación, se decide realizar un nuevo análisis de componentes principales de tipo Promax sometiendo ambos ítems al análisis, dado que sus saturaciones factoriales superaban los .4 puntos (ítem 8 $=.760 ;$ ítem $16=.662$ ); la nueva solución obtenida (que excluía a los ítems 7, 18, 15, 19 y 6) disponía de cuatro factores que explicaban el $51.280 \%$ de la varianza acumulada $(K M O=$ $\left..647 ; X^{2}=534.947 ; g l=91 ; p=.000\right)$, arrojando ahora cargas factoriales inferiores a 4 puntos para los ítems 8 y 16, motivo por el que tales ítems fueron excluidos provisoriamente. Acto seguido, un nuevo análisis de componentes principales con rotación Promax (eliminado los ítems $7,18,15,19,6,8$ y 16) extrajo una solución de cuatro factores que explicaban el $56.704 \%$ de la varianza total $\left(K M O=.625 ; X^{2}=439,730 ; g l=66 ; p=.000\right)$. Tales factores, fueron etiquetados conceptualmente de la siguiente forma: Factor 1: Preferencia o confianza por la intuición (ítems: 11, 12, 14); Factor 2: Rechazo por la intuición (ítems: 10, 5, 9); Factor 3: Percepción de la adecuación y efectividad decisoria (ítems: 17, 4, 1); Factor 4: Preferencia o confianza por el análisis (ítems: 2, 3, 13). La consistencia interna general para las cuatro escalas resultó baja $(\alpha=.569)$.

Finalmente, se decide realizar un último análisis de componentes principales forzando a la obtención de cinco factores, por dos motivos: intentar explicar un mayor porcentaje de varianza y alcanzar un mayor nivel de confiabilidad de la escala general contando con una mayor cantidad de ítems; este último motivo, resulta de observar que el contenido de los ítems 8 y 16 podría considerarse como representativo de una quinta dimensión teórica ausente en la solución de cuatro factores, a saber "Rechazo por el análisis", en contraste con la dimensión "Rechazo por la intuición".

De forma tal que, reincluyendo los ítems 8 y 16 en un último análisis de componentes 
principales, se obtiene la siguiente solución factorial definitiva: se alcanza un valor aceptable de KMO de .647, indicando nuevamente que la matriz de datos resulta factorizable; la prueba de esfericidad de Bartlett muestra que las correlaciones de los ítems se deberían a la existencia de los factores latentes extraídos $\left(X^{2}=534.947 ; g l=91 ; p=.000\right)$, difiriendo de la solución de cuatro factores (12 ítems), que explicaba un menor porcentaje de varianza. En efecto, cuando la anterior solución arrojaba un valor de $\alpha$ de .569 ( $\alpha$ parte $1=.545 ; \alpha$ parte 2 $=.531$; Test de no aditividad de Tukey: $F=23.608 ; p=.000$ ), se observa que la solución de cinco factores alcanza un nivel de fiabilidad superior arrojando un valor de $\alpha$ de .624 ( $\alpha$ parte $1=.459 ; \alpha$ parte $2=.464 ;$ Test de no aditividad de Tukey: $F=23.442 ; p=.000)$. De todas formas, la consistencia interna de la escala continúa siendo baja.

La anterior solución provee de cinco factores (14 ítems) que explican el 58.301\% de la varianza acumulada, lo cual resulta meritorio, explicando cada factor un $17.93 \%$, un $13.684 \%$, un $11.425 \%$, un $8.240 \%$ y un $7.022 \%$, respectivamente. Se observa entonces que el porcentaje de variabilidad de cada variable original, que es explicado conjuntamente por la totalidad de los factores extraídos, resulta elevado en todos los casos.

Tabla 1. Varianza total explicada.

\begin{tabular}{cccccccc}
\hline \multicolumn{3}{c}{ Autovalores iniciales } & \multicolumn{2}{c}{$\begin{array}{c}\text { Sumas de las saturaciones al } \\
\text { cuadrado de la extracción }\end{array}$} & \multicolumn{2}{c}{$\begin{array}{c}\text { Suma de las saturaciones } \\
\text { al cuadrado de la rotación }\end{array}$} \\
Componente & Total & $\begin{array}{c}\text { \% de la } \\
\text { varianza }\end{array}$ & $\begin{array}{c}\% \\
\text { acumulado }\end{array}$ & Total & $\begin{array}{c}\text { \% de la } \\
\text { varianza }\end{array}$ & $\begin{array}{c}\% \\
\text { acumulado }\end{array}$ & Total \\
\hline 1 & 2.51 & 17.93 & 17.93 & 2.51 & 17.93 & 17.93 & 1.983 \\
2 & 1.916 & 13.684 & 31.614 & 1.916 & 13.684 & 31.614 & 1.83 \\
3 & 1.6 & 11.425 & 43.04 & 1.6 & 11.425 & 43.04 & 1.855 \\
4 & 1.154 & 8.24 & 51.28 & 1.154 & 8.24 & 51.28 & 1.711 \\
5 & .983 & 7.022 & 58.301 & 0.983 & 7.022 & 58.301 & \\
6 & .893 & 6.376 & 64.677 & & & & \\
7 & .854 & 6.1 & 70.777 & & & & \\
8 & .778 & 5.555 & 76.332 & & & & \\
9 & .69 & 4.93 & 81.262 & & & & \\
10 & .668 & 4.774 & 86.037 & & & & \\
11 & .544 & 3.887 & 89.924 & & & & \\
12 & .527 & 3.762 & 93.686 & & & & \\
13 & .461 & 3.295 & 96.981 & & & & \\
14 & .423 & 3.019 & 100 & & & & \\
\hline
\end{tabular}


Tabla 2.

Matriz de Configuración.

ETDO 11: Cuando tomo una decisión importante, generalmente confío en mis propias sensaciones viscerales, más que en reglamentos o documentos, artículos u otra información formal.

ETDO 12: Al momento de tomar decisiones importantes, confío plenamente en mi sentido común, mis sensaciones y mi intuición.

ETDO 14: Cuando enfrento una nueva decisión importante, estudio la naturaleza e implicancias resultantes de ésta, pero al momento de decidir, sigo mi propio sentido común.

ETDO 9: La experiencia no es realmente "el mejor maestro" para tomar decisiones adecuadas.

ETDO 5: Usualmente me equivoco cuando utilizo el sentido común y la intuición al tomar decisiones.

ETDO 10: En caso de duda sobre una decisión importante que debo tomar, tiendo a no apelar a mi sentido común y a mi intuición.

ETDO 4: Siento que tengo una vasta reserva de información útil a la que puedo recurrir para tomar decisiones adecuadas.

ETDO 17: Siento que tengo una buena comprensión de las situaciones, que me permite resolverlas mediante decisiones adecuadas.

ETDO 1: Cuando utilizo mi sentido común y mi intuición al momento de tomar decisiones importantes, usualmente éstas resultan correctas en relación a los objetivos planteados.

ETDO 2: Al momento de tomar decisiones, tengo una gran confianza en informes o reglamentos, hechos reales y demás informaciones concretas.

ETDO 13: Yo he encontrado que confiar en información formal tal como reportes, artículos y gráficos o esquemas, en lugar de recurrir a mi sentido común, es el mejor recurso para tomar una decisión.

ETDO 3: Generalmente confío en hechos y cifras más que en mi intuición cuando tengo que tomar una decisión importante.

ETDO 16: Confiando demasiado en hechos y cifras, a menudo las decisiones resultan inadecuadas o poco realistas.

ETDO 8: Con frecuencia, tomo importantes decisiones sin recurrir ampliamente al uso de reportes escritos, documentos normativos, hechos previos, cifras e información concreta.

Método de extracción: Análsis de componentes principales. Método de rotación: Normalización de Promax con Kaiser.

a. La rotación ha convergido en 5 iteraciones.

\begin{tabular}{|c|c|c|c|c|}
\hline \multicolumn{5}{|c|}{ Componente } \\
\hline 1 & 2 & 3 & 4 & 5 \\
\hline .856 & -.049 & -.294 & .030 & .130 \\
\hline .715 & -.074 & .178 & .003 & -.048 \\
\hline .649 & .087 & .169 & -.034 & -.008 \\
\hline .089 & .766 & .075 & .013 & -.239 \\
\hline-.175 & .721 & -.021 & .037 & .195 \\
\hline .027 & .720 & -.082 & -.020 & .089 \\
\hline-.026 & .067 & .726 & -.004 & -.029 \\
\hline .035 & .047 & .721 & -.025 & .088 \\
\hline .009 & -.166 & .669 & .047 & .059 \\
\hline .083 & -.013 & .094 & .806 & .053 \\
\hline .034 & .082 & -.048 & .709 & -.251 \\
\hline-.120 & -.037 & -.041 & .696 & .190 \\
\hline-.023 & -.086 & .052 & .028 & .837 \\
\hline .173 & .229 & .051 & -.034 & .563 \\
\hline
\end{tabular}


En función de las cargas factoriales extraídas, los cinco factores definitivos de la Escala de Toma de Decisiones Operativas (ETDO) ( $\alpha=.624)$ y sus respectivos ítems, son los siguientes:

Componente 1. Preferencia o confianza por la intuición (ítems: 11, 12, 14) $(\alpha=.633)$. Cuando tomo una decisión importante, generalmente confío en mis propias sensaciones viscerales, más que en reglamentos o documentos, artículos u otra información formal (.856); Al momento de tomar decisiones importantes, confío plenamente en mi sentido común, mis sensaciones y mi intuición (.715); Cuando enfrento una nueva decisión importante, estudio la naturaleza e implicancias resultantes de ésta, pero al momento de decidir, sigo mi propio sentido común (.649).

Componente 2: Rechazo por la intuición (ítems: 10, 5, 9) $(\alpha=.609)$. La experiencia no es realmente "el mejor maestro" para tomar decisiones adecuadas (.766); Usualmente me equivoco cuando utilizo el sentido común y la intuición al tomar decisiones (.721); En caso de duda sobre una decisión importante que debo tomar, tiendo a no apelar a mi sentido común y a mi intuición (.720).

Componente 3: Percepción de la adecuación y efectividad decisoria (ítems: $17,4,1)(\alpha=$ .551). Siento que tengo una vasta reserva de información útil a la que puedo recurrir para tomar decisiones adecuadas (.726); Siento que tengo una buena comprensión de las situaciones, que me permite resolverlas mediante decisiones adecuadas (.721); Cuando utilizo mi sentido común y mi intuición al momento de tomar decisiones importantes, usualmente éstas resultan correctas en relación a los objetivos planteados (.669).

Componente 4: Preferencia o confianza por el análisis (ítems: $2,13,3)(\alpha=.589)$. Al momento de tomar decisiones, tengo una gran confianza en informes o reglamentos, hechos reales y demás informaciones concretas (.806); Yo he encontrado que confiar en información formal tal como reportes, artículos y gráficos o esquemas, en lugar de recurrir a mi sentido común, es el mejor recurso para tomar una decisión (.709); Generalmente confío en hechos y cifras más que en mi intuición cuando tengo que tomar una decisión importante (.696).

Componente 5: Rechazo por el análisis (ítems: 16, 8) $(\alpha=.415)$. Confiando demasiado en hechos y cifras, a menudo las decisiones resultan inadecuadas o poco realistas (.837); Con frecuencia, tomo importantes decisiones sin recurrir ampliamente al uso de reportes escritos, documentos normativos, hechos previos, cifras e información concreta (.563). 


\section{Discusión}

En diversos ámbitos organizacionales (e.g. empresariales, militares, entre otros), la efectividad profesional suele ser obstaculizada cuando el conocimiento acumulativo (assimilative knowledge; Kolb, 1984) coloca a la racionalidad como un recurso incuestionable (Paparone \& Reed, 2008); este tipo de conocimiento (propio de las organizaciones burocráticas) reúne ciertas tareas y estándares de trabajo que se estructuran rutinariamente, materializándose en registros escritos, reglamentos, doctrinas, lecciones aprendidas, programas de instrucción y otros datos que promueven la modificación de roles, normas y valores dentro de la comunidad. En la actualidad, tal transformación se alcanza, en gran medida, a través de tecnología institucionalizada, mecanizando determinados patrones decisorios pre-establecidos, difícilmente adaptables a contextos de crisis e incertidumbre (e.g. cultural, ambiental, entre otros; Azzollini, Torres, Depaula, Clotet, \& Nistal, 2010a; Azzollini, Torres, Depaula, Clotet, \& Nistal, 2010b).

Volviendo a las diferentes perspectivas teóricas que pretenden dar luz a las eventualidades, ambigüedades y limitaciones subyacentes a los fenómenos, procesos y modelos de toma de decisiones, Sadler-Smith y Sparrow (2008), apoyándose en la idea de Simon (1982) acerca de una racionalidad limitada, argumentan que la intuición corresponde a la voluntad de decidir cuándo los hechos o datos disponibles son escasos para inferir deliberadamente acerca de las relaciones que existen entre la planificación u objetivos iniciales y la suma de causas y efectos que constituyen el fenómeno sobre el que actuará el decisor, existiendo cierta complementariedad entre procesos analíticos e intuitivos. En línea con el concepto de sensemaking, es decir el uso del sentido común al momento de tomar decisiones (Weick, 1995), Dane y Pratt (2007) señalan que las intuiciones corresponden a juicios cargados de afecto que se originan a través de asociaciones rápidas, no conscientes y holísticas. En consonancia con lo antedicho, Klein (2003) argumenta que las decisiones más efectivas tomadas por bomberos y enfermeros, por ejemplo, derivan de este tipo de asociaciones constituidas por experiencias que se agrupan de modo inconsciente bajo la forma de patrones de reconocimiento (de acuerdo al modelo teórico denominado Recognition-Primed Decision, RPD).

En síntesis, el presente estudio aporta la adaptación de un instrumento para la evaluación de estilos decisorios desplegados frente a ciertas tareas o situaciones que enfrentan los sujetos evaluados, cuando cotidianamente toman decisiones en el marco de sus 
acciones socio-profesionales $\mathrm{u}$ ocupacionales. Sus propiedades psicométricas resultan aceptables, pero particularmente, los autores reconocen significativos obstáculos resultantes del análisis de la consistencia interna, es decir los bajos niveles de confiabilidad global y dimensional, al tiempo que no se ha analizado la validez de criterio (concurrente y predictiva). Esta última limitación representa una pretensión para futuros estudios.

Considerando críticamente las bajas confiabilidades alcanzadas en el análisis de la ETDO, tanto para la escala global como para las cinco dimensiones de este cuestionario, los autores coinciden con las ideas de Hardin y Leong (2004) en relación al instrumento DMI, suponiendo que este problema resulta de que las sub-escalas se componen de solo tres ítems; más aún el caso de la dimensión Rechazo por el análisis, compuesta por solo dos ítems, la que presentó el nivel de fiabilidad parcial más bajo $(\alpha=.415)$. No obstante, los autores del presente estudio sostienen la posibilidad de que la escasa fiabilidad parcial y global de la escala se deba a cierta precariedad en el uso de items redundantes, es decir ítems de contenido similar que exploren una misma dimensión; si bien una mayor cantidad de reactivos podría asegurar confiabilidades meritorias, numerosas pruebas disponen de una gran cantidad de ítems o frases muy parecidas, lo que demanda una mayor cantidad de tiempo para completar el cuestionario, pudiendo generar ansiedad, rechazo por la prueba o disminución de la intensidad atencional del sujeto evaluado. No es el caso del instrumento ETDO, puesto que los escasos ítems de cada sub-escala, no muestran similitudes de contenido más que las necesarias para reactivar cada aspecto decisorio propio de la dimensión conceptual que operacionalizan.

Otra de las posibles dificultades evidenciadas en este instrumento, que podría afectar su fiabilidad, corresponde al uso de ítems integrados por una gran cantidad de palabras (e.g., ítems 3, 14 y 1). En general, la teoría clásica de los test sugiere que la estructura del ítem no supere un número de veinte palabras; pero para la ETDO, al tratarse de una traducción/adaptación, se intentó respetar las frases evitando modificar demasiado el instrumento de origen.

Más allá de las precariedades del estudio mencionadas arriba, la posibilidad que brinda la ETDO de evaluar el modo en que las personas habitualmente toman sus decisiones, permitirá transferir los perfiles resultantes en programas de educación o entrenamiento de la intuición (Sadler-Smith \& Burke, 2009) aplicados a solados, suboficiales u oficiales militares (que ejercen diferentes roles de liderazgo), los cuales se enfrentan a ambientes de gran 
incertidumbre propios de los actuales escenarios de accionar militar (Azzollini, Torres, \& Depaula, 2010; Mancini, Clotet, \& Depaula, 2010), así como en ámbitos empresariales donde deban tomarse decisiones rápidas con escasa información.

La evaluación de los procesos de toma de decisiones en el ámbito de la educación alcanza un significativo nivel de relevancia social, debiendo estos ser considerados como procesos dinámicos y flexibles para responder a los cambios que emergen en los diversos niveles de formación, en términos de los contextos de aplicabilidad, de la diversidad de cursantes y profesionales -potenciales decisores- y de la revisión y mejora de los contenidos curriculares (e.g., de capacitación profesional, de entrenamiento, etc.) (Woods-Groves \& Hendrickson, 2012). Por estas cuestiones, sería interesante expandir las posibilidades de generalizar los resultados analizando las propiedades psicométricas de la ETDO en muestras de estudiantes y profesionales que operan en ámbitos civiles.

En general, algunos autores coinciden en que los estilos de toma de decisiones y de pensamiento no resultan significativamente afectados por variables tales como el género, la edad o el status ocupado en el lugar de trabajo (Antonietti et al., 2005; Raffaldi, et al., 2012), puesto que su estabilidad se debe a características individuales profundamente arraigadas; por ello, acuerdan en que los instrumentos que evalúan tales estilos pueden ser utilizados con facilidad y consistencia en procesos de selección y entrenamiento de recursos humanos, sobre todo porque requieren de breves tiempos de administración y de análisis de los puntajes escalares. No obstante, como se adelantó en anteriores párrafos, los autores reconocen que los cuestionarios de auto-reporte presentan obstáculos si se considera la posibilidad de que los aspectos o variables situacionales influyan en la emergencia o el despliegue de diferentes estilos decisorios, frente a lo novedoso del escenario de trabajo actual donde operan los decisores.

Curseu y Schruijer (2012; Curseu, 2011) evidencian el carácter distintivo de los estilos de toma de decisiones relacionados con competencias decisorias específicas, reconocen que aún no han podido explicar claramente si los estilos decisorios predicen la búsqueda de información en diversas situaciones decisorias $\mathrm{y}$, concomitantemente, argumentan que la diversidad de decisiones se arraigan en la amplitud y complejidad de los contextos sociales; por ello, sugieren desarrollar un mayor número de investigaciones que exploren las implicancias de los estilos decisorios sobre la búsqueda de información en diferentes escenarios sociales (Azzollini, Torres, \& Depaula, 2010; Azzollini, Torres, 
Depaula, Clotet, \& Nistal, 2010a,b; Curseu, 2011, 2012). Bajo este panorama, se justifica la especificidad de la muestra seleccionada, ya que resulta de interés estudiar los estilos decisorios de los cascos azules, puesto que operan en ambientes culturales diversos, en tanto su decisiones estarían influenciadas por estos patrones culturales que difieren a los de su propia cultura. En consonancia con Hardin y Leong (2004), los autores de este estudio sugieren realizar futuras investigaciones que analicen la efectividad de las intervenciones profesionales conforme a las diferencias individuales en materia de estilos de toma de decisiones y las singularidades situacionales de los ambientes o contextos sociales decisorios.

Cabe señalar que resultaría enriquecedor analizar la ETDO a través de la generación de un modelo de ecuaciones estructurales a fin de testear la validez de la escala en relación al modelo teórico subyacente. Por otro lado, sería interesante avanzar en la construcción y validación empírica de un nuevo instrumento que facilite la evaluación de los procesos de toma de decisiones, focalizándose particularmente en un modelo multidimensional que contraste mecanismos cognitivos de detección de rasgos o patrones de reconocimiento, visuales sobre todo (Boyd, 1987; Bryant, 2006; Zimmerman et al., 2011), comparando modelos decisorios reactivos y proactivos, evaluando la cognición dirigida a objetivos (Hatano \& Inagaki, 2000) versus la orientación y adaptación rápida a los cambios circunstanciales del ambiente (Scott, McCone, \& Mastroianni, 2009).

Finalmente, las dificultades encontradas en la aplicación de pruebas psicométricas para evaluar los estilos de toma de decisiones, sugieren una posible solución en la recolección de otro tipo de información (e.g., tratamientos experimentales mediante el uso de imágenes estáticas o dinámicas -fotografías o videos-) en la que se proponga un acercamiento más concreto, específico y directo en relación al mundo del trabajo, describiendo el decisor su propia conducta frente a una situación/problema por resolver que revista cierto contenido realista a través de la simulación (Depaula et al., 2012; Raffaldi et al., 2012), ya sea en contextos militares como en el ámbito universitario o de los negocios.

\section{Referencias}

Aczel, B., Lukacs, B., Komlos, J., \& Aitken, M R. F. (2011). Unconscious intuition or conscious analysis? Critical questions for the Deliberation-Without-Attention paradigm. Judgment and Decision Making, 6(4), 351-358. 
Aiken, L. R. (2002). Psychological testing and assessment. New York: Allyn \& Bacon.

Antonietti, A., Fabio, R. A., Boari, G., \& Bonanomi, A. (2005). The questionnaire "Style of Learning and Thinking" (SOLAT): Psychometric data, validation, and standardization of the Italian version. TPM Testing-Psicometria-Metodologia, 12, 299-316.

Atay, S. (2012). The Standardization of Myers-Briggs Type Indicator into Turkish: An Application on Students. Journal of Instructional Psychology, 39(2), 74-79.

Azzollini, S. C., Depaula, P. D., Piñeyro, D. R., \& Torres, J. A. (2012). Incidencia del estilo decisorio predominante y el grado de incertidumbre cultural en la efectividad de las decisiones tomadas por militares voluntarios en misiones de paz. Revista Argentina de Ciencias del Comportamiento, 4(3), 13-21.

Azzollini, S., Torres, A., Depaula, P., Clotet, C., \& Nistal, M. (2010a). La tensión entre la adaptación y la extrañeza cultural: Los significados atribuidos por integrantes de misiones de paz a los hábitos y costumbres de los lugareños. VI Jornadas de Sociología de la UNLP, "Debates y perspectivas sobre Argentina y América Latina en el marco del Bicentenario. Reflexiones desde las Ciencias Sociales”. Departamento de Sociología de la Facultad de Humanidades y Ciencias de la Educación de la Universidad Nacional de La Plata (UNLP). (pp. 1-11). (ISBN 978950-34-0693-9).

Azzollini, S., Torres, A., Depaula, P., Clotet, C., \& Nistal, M. (2010b). Los significados atribuidos a las situaciones de incertidumbre por militares integrantes de una misión de paz en Haití. VI Jornadas de Etnografía y Métodos Cualitativos, organizadas por el Instituto de Desarrollo Económico y Social (IDES). Centro de Antropología Social (CAS). (pp. 1-9). (ISBN 978-987-23365-2-3).

Azzollini, S., Torres, J. A., \& Depaula, P. (2010). Los procesos de toma de decisiones en los escenarios militares actuales. Congreso Panamericano de Psicología 2010, "Abordajes psicológicos ante los emergentes de la sociedad actual". Departamento de Psicología de la Facultad de Artes y Ciencias de la Universidad Católica de Salta (UCASAL). (pp. 1-11). (ISBN 978-950-623-060-9).

Betsch, C. (2004). Preference for intuition and deliberation (PID): An inventorying for assessing affect- and cognition-based decisionmaking. Zeitschrift für Differentielle und Diagnostische Psychologie, 25, 179-197.

Betsch, C. (2007). Chronic preferences for intuition and deliberation in decision making. En: H. Plessner, C. Betsch, \& T. Betsch (Eds.), Intuition in judgment and decision making 
(pp. 3-22). Mahwah: Lawrence Erlbaum.

Betsch, C., \& Kunz, J. J. (2008). Individual Strategy Preferences and Decisional Fit. Journal of Behavioral Decision Making, 21(5), 532-555

Boyd, J. R. (1987). A discourse on winning and losing [Briefing slides]. Maxwell Air Force Base, AL: Air University Library. (Document No. M-U 43947).

Briggs Myers, I., \& Myers, P. B. (1980). Gifts differing. Palo Alto: Consulting Psychologists Press.

Bryant, D. J. (2006). Rethinking OODA: Toward a Modern Cognitive Framework of Command Decision Making. Military Psychology, 18(3), 183-206.

Coscarelli, W. C. (1983). Development of a decision making inventory to assess Johnson's decision making styles. Measurement and Evaluation in Guidance, 16, 149-160.

Curseu, P. L. (2011). Need for cognition and active information search in small student groups. Learning and Individual Differences, 21, 415-418.

Curseu, P. L., Schruijer, G. L. (2012). Decision Styles and Rationality: An Analysis of the Predictive Validity of the General Decision-Making Style Inventory. Educational and Psychological, 72(6), 1053-1062.

Daake, D., Dawley, D. D., \& Anthony, W. P. (2004). Formal Data Use in Strategic Planning: An Organizational Field Experiment. Journal of Management Issues, 16(2), 232-247.

Dane, E., \& Pratt, M. G. (2007). Exploring intuition and its role in managerial decisionmaking. Academy of Management Review, 32(1), 33-54.

Demir, Y., Denat, Y., Khorshid, L., \& Eser, I. (2012). Cultural Validation of the "Use of Intuition by Nursing Students Scale - Turkish versión”. Journal of Transcultural Nursing, 23(4), 369-376.

Depaula, P., Torres, J. A., Piñeyro, D. R., Cosentino, A. C., Clotet, C. I., \& Castillo, S. (2012). Desarrollo de un software para la evaluación de la toma de decisiones en contextos de incertidumbre cultural. Memorias IV Congreso Internacional de Investigación y Práctica Profesional en Psicología, XIX Jornadas de Investigación, VIII Encuentro de Investigadores en Psicología del MERCOSUR. Secretaría de Investigaciones de la Facultad de Psicología de la Universidad de Buenos Aires (UBA). Neuropsicología y Psicología Cognitiva. (p. 67). (ISSN 1667-6750).

Dijksterhuis, A., \& Nordgren, L. F. (2006). A theory of unconscious thought. Perspectives on Psychological Science, 1, 95-109. 
Epstein, S. (1994). Integration of the cognitive and the psychodynamic unconscious. American Psychologist, 49, 709-724.

Epstein, S. (2007). Intuition from the perspective of cognitive-experiential self-theory. En: H. Plessner, C. Betsch, \& T. Betsch (Eds.), Intuition in judgment and decision making (pp. 23-37). Mahwah: Lawrence Erlbaum.

Evans, J. S. B. T. (2008). Dual-processing accounts of reasoning, judgment, and social cognition. Annual Review of Psychology, 59, 255-278.

Gregory, R. J. (2007). Psychological testing: History, principles, and applications. Boston: Pearson Education.

Hardin, E. E. \& Leong, F. T. L. (2004). Decision-Making Theories and Career Assessment: A Psychometric Evaluation of the Decision Making Inventory. Journal of Career Assessment, 12(1), 51-64.

Hatano, G., \& Inagaki, K. (2000). Knowledge acquisition and use in higher-order cognition. En: K. Pawlik \& M. R. Rosenzweig (Eds.), International handbook of psychology (p. 167-190). London: Sage.

Johnson, R. H. (1978). Individual styles of decision making: A theoretical model for counseling. Personnel and Guidance Journal, 56, 530-536.

Jung, C. (1964). Tipos psicológicos. Buenos Aires: Sudamericana.

Kahneman, D. (2003). A perspective on judgment and choice. Mapping bounded rationality. American Psychologist, 58, 697-720.

Klein, G. (1998). Sources of Power: How People Make Decisions. Cambridge, MA: MIT Press.

Klein, G. (2003). Intuition at work: Why developing your gut instincts will make you better at what you do. New York: Currency Doubleday.

Kolb, D. (1984). Experiential Learning: Experience as the Source of Learning and development. Englewood Cliffs, NJ: Prentice- Hall.

Mancini, J. C. M., Clotet, C. I., \& Depaula, P. (2010). Las Naciones Unidas y su aporte a la paz mundial. Congreso Internacional "Profundizando la democracia como forma de vida. Desafíos de la democracia participativa y los aprendizajes ciudadanos en el Siglo XXI”. Facultad de Ciencia Política y Relaciones Internacionales de la Universidad Nacional de Rosario (UNR). (pp. 302-306). (ISBN 978-950-673-868-6).

Mintzberg, H. (1973). The nature of managerial work. New York: Harper \& Row. 
Nisbett, R. E., Peng, K., Choi, I., \& Norenzayan, A. (2001). Culture and systems of thought: Holistic vs. analytic cognition, Psychological Review, 108, 291-310.

Paparone, C. R., \& G. Reed (2008). The Reflective Military Practioner: How Military Professionals Think in Action. Military Review, 88(2), 66-76.

Pritchard, J. (2009). Tools for supporting intuition. Coaching: An International Journal of Theory, Research and Practice, 2(1), 37-43.

Raffaldi, S., Iannello, P., Vittani, L., \& Antonietti, A. (2012). Decision-Making Styles in the Workplace: Relationships Between Self-Report Questionnaires and a Contextualized Measure of the Analytical-Systematic Versus Global-Intuitive Approach. Sage Open, 2, 1-11.

Riding, R., \& Rayner, S. (1998). Cognitive styles and learning strategies. London: Fulton.

Sadler-Smith, E., \& Burke, L. A. (2009). Fostering Intuition in Management Education: Activities and Resources. Journal of Management Education, 33, 239-262.

Sadler-Smith, E., \& Sparrow, P. R. (2008). Intuition in Organizational Decision Making. En G. P. Hodgkinson, \& W. H. Starbuck (Eds.), The Oxford Handbook of Organizational Decision Making (pp. 305-324). New York: Oxford University Press.

Scott, S. G., \& Bruce, R. A. (1995). Decision-making style: The development of a new measure. Educational and Psychological Measurement, 55, 818-831.

Scott, W. J., McCone, D. R., \& Mastroianni, G. R. (2009). Armed Forces \& Society, 35(3), 460-476.

Simon, H. (1947). Administrative Behavior. Nueva York: Macmillan.

Simon, H. (1982). The Sciences of The Artificial. Cambridge, MA: MIT Press.

Simon, H. (1987). Making Management Decisions: The Role of Intuition and Emotion. Academy of Management Executive, 1(1), 57-66.

Simon, H. A. (1955). A behavioral model of rational choice. The Quarterly Journal of Economics, 69, 99-118.

Smith, A. J. (2003). The development and psychometric testing of an instrument measuring the use of intuition by nursing students. Unpublished doctoral dissertation, University of San Diego, San Diego, CA.

Smith, A. J. (2006). Continued Psychometric Evaluation of an Intuition Instrument for Nursing Students. Journal of Holistic Nursing, 24(2), 82-89.

Smith, A. J., Thurkettle, M. A., \& dela Cruz, F. A. (2004). Use of intuition by nursing 
students: Instrument development and testing. Journal of Advanced Nursing, 47(6), 614-622.

Torrance, E. P. (1987). Some evidence regarding development of cerebral lateralization. Perceptual and Motor Skills, 64, 261-262.

Weick, K. E. (1995). Sensemaking in Organizations. Thousand Oaks, CA: Sage.

Woods-Groves, S., \& Hendrickson, J. M. (2012). The Role of Assessment in Informing Our Decision-Making Processes. Assessment for Effective Intervention, 38(1), 3-5.

Zimmerman, L. A., Mueller, S. T., \& Marcon, J. L. (2011). Improving Soldier Threat Detection Skills in the Operational Environment. Interservice/Industry Training, Simulation, and Education Conference. Paper No. 11196 (pp. 1-12).

\footnotetext{
${ }^{\text {i }}$ La mencionada investigación se desarrolla en el marco de los siguientes proyectos: PIDDEF 33/10, titulado "La toma de decisiones para la defensa en contextos diversos y de gran incertidumbre y su relación con la inteligencia cultural y los estilos de liderazgo, en militares asignados a misiones de paz", financiado por el Ministerio de Defensa; y el PIP 11220100100449, titulado "La influencia de la memoria autobiográfica y la inteligencia cultural sobre la toma de decisiones en contextos caracterizados por la diversidad cultural y la incertidumbre", cuyo organismo de financiamiento corresponde al Consejo Nacional de Investigaciones Científicas y Técnicas (CONICET).
} 